Urologe 2017·56:180-185

DOI 10.1007/s00120-016-0271-6

Online publiziert: 15 . November 2016

(c) Der/die Autor(en) 2016. Dieser Artikel ist eine Open-Access-Publikation.

CrossMark

\author{
H. Braunwarth $\cdot$ F. H. H. Brill ${ }^{2}$ J. Steinmann ${ }^{4}$ D. Hegeholz $\cdot$ W. Droste ${ }^{3}$ \\ ${ }^{1}$ Coloplast GmbH, Hamburg, Deutschland \\ ${ }^{2}$ Dr. Brill + Partner GmbH Institut für Hygiene und Mikrobiologie, Hamburg, Deutschland \\ ${ }^{3}$ Selm, Deutschland \\ ${ }^{4}$ Institut für Medizinische Mikrobiologie, Universitätsklinikum Essen, Essen, Deutschland
}

\section{Wanderungsgeschwindigkeit von uropathogenen Erregern}

\section{Bestimmung im Ureterkatheter im praxisnahen In-vitro-Modell}

stoßen der Rücklaufsperre. Außerdem werden nicht nur bei Ileumconduits, sondern auch bei anderen Formen der supravesikalen Harnableitung temporär Harnleiterschienen eingelegt. Da diese auch durch das für die Harnableitung verwendete Darmstück gezogen werden, ist eine Kontamination auch bei intakter Rücklaufsperre die Regel.

Ziel unserer In-vitro-Untersuchungen war es, die Wanderungs- bzw. Wachstumsgeschwindigkeit von klinisch relevanten Bakterien durch das harnableitende System zu untersuchen. Hiermit sollte geprüft werden, wie wahrscheinlich es ist, dass Bakterien aufgrund einer
Kontamination, z. B. aus einem kontaminierten Urinbeutel oder Katheter die Niere innerhalb der Liegedauer der Splintkatheter von bis zu 14 Tagen erreichen, um dort evtl. eine Infektion auslösen $\mathrm{zu}$ können.

\section{Material und Methoden}

Alle Versuche wurden unter einer Reinraumwerkbank durchgeführt, um Kontaminationen über die Luft auszuschließen. Ein Vorratsgefäß wurde über die bei der Harnleiterimplantation üblichen Splintkatheter (Splints Coloplast Porges ACA107, Ch/Fr07/2.3 mm Vortek single mit $33-63 \%$ und weltweit die derze häufigste Form der Harnableitung nach operativer Entfernung der Harnblase. In den Fällen, in denen die Anlage eines Ileumconduit nicht durchgeführt werden kann, erfolgt in den meisten Fällen die Ableitung des Harns über die Ureterhautfistel [9]. Für 10-14 Tage wird nach diesen Operationen in der Heilungsphase der Harn über Splintkatheter in einen Urinbeutel abgeführt. Diese Splintkatheter werden dann bei komplikationslosem Verlauf entfernt [2]. Bei Auftreten von Komplikationen kann dieser Zeitraum verlängert werden. Das Einlegen von Splintkathetern während der Operation ist die übliche Vorgehensweise und der Nutzen durch Studien ausreichend belegt [3]. Es ist nicht auszuschließen, dass in der Praxis die Splintkatheter länger als 10-14 Tage liegen und mit ggf. kontaminiertem Harn im Beutel in Kontakt kommen können, z. B. beim Durch-

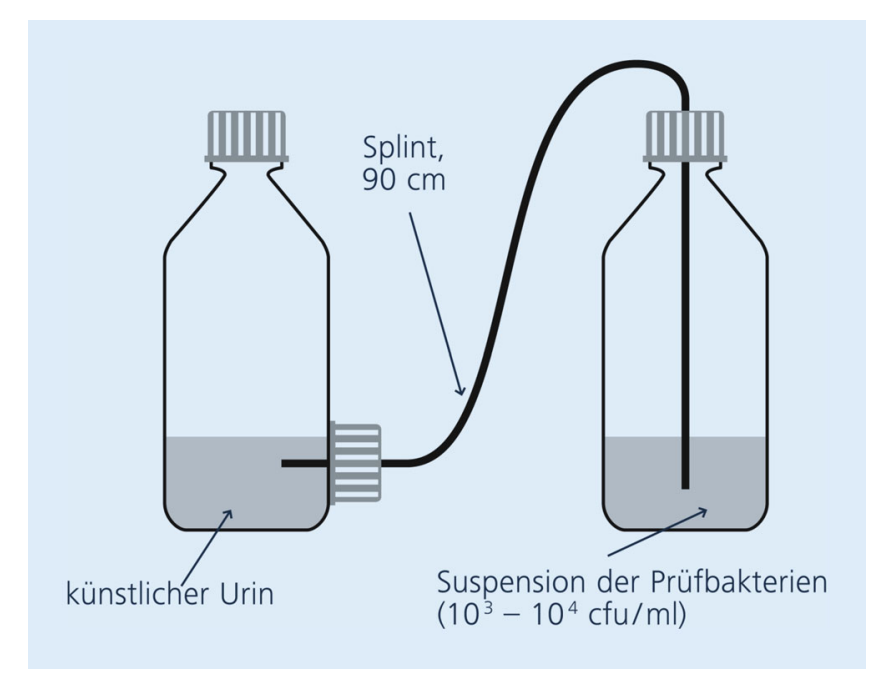

Abb. 1 \ Versuchsaufbau: Ein Vorratsgefäß wurde über die bei der Urostomaversorgung üblichen Katheter (Splints, Länge $90 \mathrm{~cm}$ ), die vorher einmalig mit künstlichem Harn gespült wurden, mit einem zweiten Vorratsgefäßverbunden. Im Vorratsgefäß wurde die Bakteriensuspension in künstlichem Harn vorgelegt. Die beiden Vorratsgefäße wurden in einem Brutschrank bei $36^{\circ} \mathrm{C}, 24-72 \mathrm{~h}$ unter leichtem Schütteln bebrütet 
Hier steht eine Anzeige.

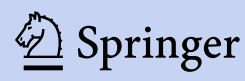


Tab. 1 Mittelwerte und Standardabweichungen der Wanderungsstrecken von E. coli, S. aureus und P. mirabilis nach 24,48 und $72 \mathrm{~h}$ im Splint

\begin{tabular}{|l|lllllll} 
& E. coli & \multicolumn{3}{c}{ S. aureus } & P. mirabilis \\
& cm & SD & cm & SD & cm & SD \\
\hline $24 \mathrm{~h}$ & 26,7 & 20,6 & 27,2 & 10,6 & 9,4 & 14,0 \\
\hline $48 \mathrm{~h}$ & 36,1 & 12,7 & 51,7 & 7,5 & 30,6 & 21,3 \\
\hline $72 \mathrm{~h}$ & 45,6 & 11,0 & 57,5 & 15,1 & 67,8 & 3,6 \\
\hline
\end{tabular}

SD Standardabweichung

Tab. 2 Ergebnis der statistischen Prüfung auf Unterschiede der Mittelwerte der Wanderungsstrecken von E. coli, S. aureus und P. mirabilis im t-Test. Angegeben sind die $p$-Werte, signifikante statistische Unterschiede sind fett hervorgehoben

\begin{tabular}{llll} 
& E. coli & S. aureus & P. mirabilis \\
\hline $24 \mathrm{~h} / 48 \mathrm{~h}$ & $p=0,26$ & $\boldsymbol{p}<0,001$ & $\boldsymbol{p}<0,01$ \\
\hline $48 \mathrm{~h} / 72 \mathrm{~h}$ & $\boldsymbol{p}=0,03$ & $p=0,18$ & $\boldsymbol{p}=0,01$ \\
$24 \mathrm{~h} / 72 \mathrm{~h}$ & $\boldsymbol{p}=0,02$ & $\boldsymbol{p}<0,001$ & $\boldsymbol{p}<0,001$
\end{tabular}

Tab. 3 Ergebnis der statistischen Prüfung auf Unterschiede der Mittelwerte der Wanderungsstrecken von $E$. coli, S. aureus und $P$. mirabilis nach 24,48 und $72 \mathrm{~h}$ im t-Test. Angegeben sind die $p$-Werte, signifikante statistische Unterschiede sind fett hervorgehoben

\begin{tabular}{llllllll} 
& $\mathbf{2 4} \mathbf{h}$ & \multicolumn{3}{c}{$\mathbf{4 8} \mathbf{h}$} & $\mathbf{7 2} \mathbf{h}$ \\
& E. coli & S. aureus & E. coli & S. aureus & E. coli & S. aureus \\
\hline S. aureus & $p=0,94$ & - & $p<0,01$ & - & $p=0,16$ & - \\
P. mirabilis & $p=0,13$ & $\boldsymbol{p}=\mathbf{0 , 0 4}$ & $p=0,46$ & $p=0,25$ & $\boldsymbol{p}<0,01$ & $p=0,17$
\end{tabular}

loop ureteral stent, open renal tip drainage, Länge $90 \mathrm{~cm}$ ), die vorher einmalig mit künstlichem Harn gespült wurden, mit einem zweiten Vorratsgefäß verbunden, in dem eine Bakteriensuspension der Testbakterien in künstlichem sterilen Harn vorgelegt wurde. Der Versuchsaufbau ist in - Abb. 1 dargestellt. Die beiden Vorratsgefäße wurden in einem Brutschrank bei $36^{\circ} \mathrm{C}, 24,48$ oder $72 \mathrm{~h}$ unter leichtem Schütteln bebrütet. Der künstliche Harn entsprach in seiner Zusammensetzung der DIN EN 1616:2003 [1].

Als Testbakterien wurden Escherichia coli K12 (E. coli, NCTC 10538), Methicillin-resistenter Staphylococcus aureus (MRSA, S. aureus, ATCC 33592) und Proteus mirabilis (P. mirabilis, ATCC 14153) verwendet. Alle Testkeime gehören zu häufigsten Erregern der unkomplizierten Harnwegsinfektionen die zu $80 \%$ auf E.coli zurückzuführen sind [4]. Die Ausgangskeimzahlen betrugen ca. $10^{4}$ koloniebildende Einheiten $(\mathrm{KBE}) / \mathrm{ml}$ (bei 24 und $48 \mathrm{~h}$ Bebrütung) bzw. $10^{3} \mathrm{KBE} / \mathrm{ml}$ (bei $72 \mathrm{~h}$ Bebrütung).

Nach 24, 48 und $72 \mathrm{~h}$ wurden die Splintkatheter in ganzer Länge in je $5 \mathrm{~cm}$ lange Abschnitte zerteilt und die Ab- schnitte auf Caseinpepton-Sojamehlpepon-Agar (CSA) für $24 \mathrm{~h}$ bei $36^{\circ} \mathrm{C}$ bebrütet und anschließend die KBE ausgezählt. Als Maß für die Wanderungsstrecke diente der am weitesten vom Vorratsgefäß entfernte noch kolonisierte $5 \mathrm{~cm}$ Abschnitt des Splintkatheters. Jeder Versuch wurde neunmal durchgeführt. Aus den neun Versuchen wurden der arithmetische Mittelwert und die Standardabweichungen bestimmt. Die Prüfung der Mittelwerte auf statistische Unterschiede erfolgte im $\mathrm{t}$-Test.

\section{Ergebnisse}

Die Ergebnisse der Wanderungsgeschwindigkeiten für $E$. coli, $S$. aureus und P. mirabilis im Splintkatheter sind in - Tab. 1 und $\bullet$ Abb. 2 dargestellt. Die durchschnittliche Wanderungsstrecke liegt umgerechnet auf eine Stunde auf Basis der bewachsenen Strecken nach $72 \mathrm{~h}$ bei $0,63 \mathrm{~cm}$ für $E$. coli, $0,80 \mathrm{~cm}$ für $S$. aureus und $0,94 \mathrm{~cm}$ für $P$. mirabilis.

Der Vergleich der Wanderungsstrecken ergibt im t-Test bei $E$. coli eine statistische Signifikanz im Vergleich der Daten von 48 und $72 \mathrm{~h}(p=0,03)$ und bei den Daten von 24 und $72 \mathrm{~h}$ ( $p=$ 0,02; - Tab. 2). Bei S. aureus sind die Unterschiede der Wanderungsstrecken von $S$. aureus 48 und $72 \mathrm{~h}$ signifikant $(p<$ $0,001)$ und 24 und $72 \mathrm{~h}(p<0,001)$. Bei $P$. mirabilis sind alle Datensätze statistisch signifikant unterschiedlich (24 und $48 \mathrm{~h}$, $p=0,01 ; 48$ und $72 \mathrm{~h}, p=0,01$ und 24 und 72 h, $p<0,001$; - Tab. 2). Nach 72 h ist nur die Wanderungsstrecke von E. coli im Vergleich zu P. mirabilis statistisch signifikant unterschiedlich (• Tab. 3).

\section{Diskussion}

Die Ergebnisse zeigen, dass die Testbakterien P. mirabilis, S. aureus und E. coli im Splintkatheter innerhalb kurzer Zeit relativ weite Strecken wandern bzw. wachsen können. Erwartungsgemäß nimmt die Strecke mit zunehmender Zeit bei allen geprüften Bakterien zu. Die weiteste Strecke legte $P$. mirabilis nach $72 \mathrm{~h}$ zurück. Im t-Test sind nach $72 \mathrm{~h}$ (•Tab. 3) die Wanderungsstrecken von $P$. mirabilis und E. coli statistisch signifikant unterschiedlich, nicht jedoch die von P. mirabilis und S. aureus (bzw. $S$. aureus und E. coli). Dies spricht dafür, dass sowohl begeißelte und damit in Flüssigkeit mobile Bakterien wie P. mirabilis und E. coli als auch nicht begeißelte Bakterien wie $S$. aureus relativ schnell mit $0,63-0,94 \mathrm{~cm} / \mathrm{h}$ durch den Splintkatheter wachsen können. Die Ergebnisse deuten jedoch auch darauf hin, dass für eine Besiedlung des Splintkatheters eine Begeißelung nicht notwendig ist und dies zumindest in unserem Versuchsaufbau nicht entscheidend für die Besiedlungsgeschwindigkeit und Wanderungsstrecke ist. Aus Tests an biologischen Systemen ist eine oftmals größere Streubreite der Messwerte trotz einer Standardisierung bekannt, die sich auch in unserem Versuchsansatz trotz der 9-maligen Wiederholungen findet. Die Ursache hierfür ist nicht klar, doch könnten hierfür Inhomogenitäten im Kathetermaterial, die Einfluss auf das Bakterienwachstum haben, verantwortlich sein. Solche Materialinhomogenitäten können auch innerhalb einer Charge auftreten. Aufgrund der vorliegenden Daten kann dies jedoch nicht abschließend bewertet werden. 
Die unterschiedlichen Wachstumsbedingungen in vitro (Bebrütung bei $36^{\circ} \mathrm{C}$ ) und in vivo werden oftmals als limitierender Faktor für die Übertragbarkeiten der Ergebnissen aus In-vitro-Untersuchungen in den klinischen Alltag diskutiert. In unserem Versuchsaufbau wurde er kontinuierliche Harnfluss, der beim Urostoma auftritt, nicht nachgestellt, so dass die im Versuch nachgewiesenen intraluminale Wanderungsstrecken nicht unkritisch auf den klinischen Alltag übertragen werden können. Es wurden jedoch an geschlossenen harnableitenden Systemen vergleichbare Ergebnisse erzielt [8]. Bakterien können in diesem System im Harnwegkatheter intraluminal etwa 3- bis 4-mal schneller in die Harnblase wandern als extraluminal, wie frühere Untersuchungen zeigten $[8,10]$ : Für die Ausbreitung eines PseudomonasFilms wurde dabei eine Wanderungsgeschwindigkeit von $1-2 \mathrm{~cm} / \mathrm{h}$ entgegen dem Harnfluss nachgewiesen. Auch wenn diese Ergebnisse nicht auf unseren Versuchsansatz kritiklos übertragen werden können, stützen diese Ergebnisse jedoch unsere Interpretation, dass die fehlende Simulation des Harnflusses kein limitierender Faktor ist. Die Autoren konnten zudem zeigen, dass eine Antibiotikagabe die Wanderung der Bakterien nicht vollständig verhinderte, aber auf ca. $1 / 10$ verringerte $[8,10]$. Im vorgestellten Prüfmodell wird bei der geringsten Besiedlungsgeschwindigkeit von $0,63 \mathrm{~cm} / \mathrm{h}$ bereits in etwas mehr als 3 Tagen eine Gesamtstrecke von $80 \mathrm{~cm}$ bewachsen. Bei einer Liegedauer der Splintkatheter von 10-14 Tagen kann davon ausgegangen werden, dass Bakterien aus dem Urostomabeutel in die Niere gelangen, wenn der Harn kontaminiert ist, da hier nur eine Strecke von $<40 \mathrm{~cm}$ zurückgelegt werden muss.

Entwickelt sich bei Menschen mit einer solchen Urostomie eine Harnwegsinfektion, so ist die Gefahr einer aufsteigenden Infektion der Nieren besonders groß. Die chronischen Harnwegsinfektionen beim Ileumconduit werden in der Literatur mit ca. $23 \%$ angegeben, wobei nicht klar unterschieden wird zwischen den Urostomien mit und ohne liegende Splintkatheter [2].

Urologe 2017·56:180-185 DOI 10.1007/s00120-016-0271-6

(c) Der/die Autor(en) 2016. Dieser Artikel ist eine Open-Access-Publikation.

\section{H. Braunwarth · F. H. H. Brill · J. Steinmann - D. Hegeholz - W. Droste}

\section{Wanderungsgeschwindigkeit von uropathogenen Erregern. Bestimmung im Ureterkatheter im praxisnahen In-vitro-Modell}

\section{Zusammenfassung}

Hintergrund. Bei den verschiedenen Urostomien wie dem lleumconduit und der Ureterhautfistel besteht ein hohes Infektionsrisiko für die Nieren und das harnableitende System des Patienten. Deshalb ist das Vermeiden des Aufsteigens von Bakterien im künstlichen Abfluss (Splintkatheter) durch z. B. kontaminierten Harn wichtigstes Ziel der hygienischen Maßnahmen. Es sollte in vitro nachgewiesen werden, wie schnell klinisch relevante Prüfbakterien diese Splintkatheter besiedeln können.

Methode. Es wurde die Wanderungsgeschwindigkeit von Bakterien in einem handelsüblichen Splintkatheter in einem praxisnahen In-vitro-Modell untersucht. Hierzu wurde ein Vorratsgefäß mit Splintkathetern mit einem zweiten Vorratsgefäß verbunden, in dem eine Bakteriensuspension der Prüfbakterien Escherichia coli, Proteus mirabilis und Staphylococcus aureus in künstlichem Harn vorgelegt wurde. Die beiden Vorratsgefäße wurden bei $36^{\circ} \mathrm{C}$ bis zu $72 \mathrm{~h}$ inkubiert. Die Keimzahl in den Splintkathetern wurde nach $24 \mathrm{~h}, 48 \mathrm{~h}$ und $72 \mathrm{~h}$ bestimmt, um die Besiedlungsstrecke nachzuweisen.

Ergebnisse. Die durchschnittliche Wanderungsgeschwindigkeit lag bei $0,63 \mathrm{~cm} / \mathrm{h}$ für E. coli, $0,80 \mathrm{~cm} / \mathrm{h}$ für $S$. aureus und $0,94 \mathrm{~cm} / \mathrm{h}$ für $P$. mirabilis. Dies ergibt eine gemittelte Besiedlungsstrecke von $80 \mathrm{~cm}$ in ca. 3 Tagen. Schlussfolgerungen. Aus den Ergebnissen im In-vitro-Modell lässt sich eine hohe Wanderungsgeschwindigkeit der Bakterien ableiten. In vivo würde es bedeuten, dass innerhalb der üblichen Liegedauer der Splintkatheter beim Urostoma von 10-14 Tagen vollständig besiedelt wird.

Schlüsselwörter

Urostoma · Splint · Bakterien · Hygiene · Bakterienwanderung

\section{Migration speed of uropathogens. Determination in the ureteral splint in a practice-like in vitro model}

\section{Abstract}

Background. A urostomy with an ileum conduit or a skin fistula leads to a high infection risk for the kidneys and the ureter of the patient. Therefore, the prevention of retrograde colonization of the artificial drain (splint) with e. g. contaminated urine is the most important objective of infection prevention measurements. We performed an in vitro experiment to determine the migration speed of clinically relevant bacteria in a commercially available splint catheter system.

Methods. The migration speed of bacteria in commonly used splint catheters was determined in a practice-like in vitro model. Two storage vessels were connected with splints. The second vessel contained a bacterial suspension of the test bacteria Escherichia coli, Proteus mirabilis, and Staphylococcus aureus in artificial urine. The two vessels were incubated at $36^{\circ} \mathrm{C}$ for $24-72 \mathrm{~h}$. The microbial count in the catheters was determined after each experiment to investigate the migration distance.

Results. The average migration speed was $0.63 \mathrm{~cm} / \mathrm{h}$ for $E$. coli, $0.80 \mathrm{~cm} / \mathrm{h}$ for S. aureus, and $0.94 \mathrm{~cm} / \mathrm{h}$ for $P$. aeruginosa. This results in a colonization distance of approximately $80 \mathrm{~cm}$ in 3 days.

Conclusion. If the system, e. g., the stoma pouch is contaminated, it can be expected that during the common application time of a splint of 10-14 days, the complete splint will be contaminated due to the high bacteria migration speed. Consequently there is a high infection risk for kidneys and ureters. A return stop feature in the stoma pouch should minimize this risk. However, it is of upmost importance to not applying the splint through the return stop to prevent any contact with potentially contaminated urine.

\section{Keywords}

Urostomy · Splint · Bacteria · Hygiene · Bacteria migration speed 


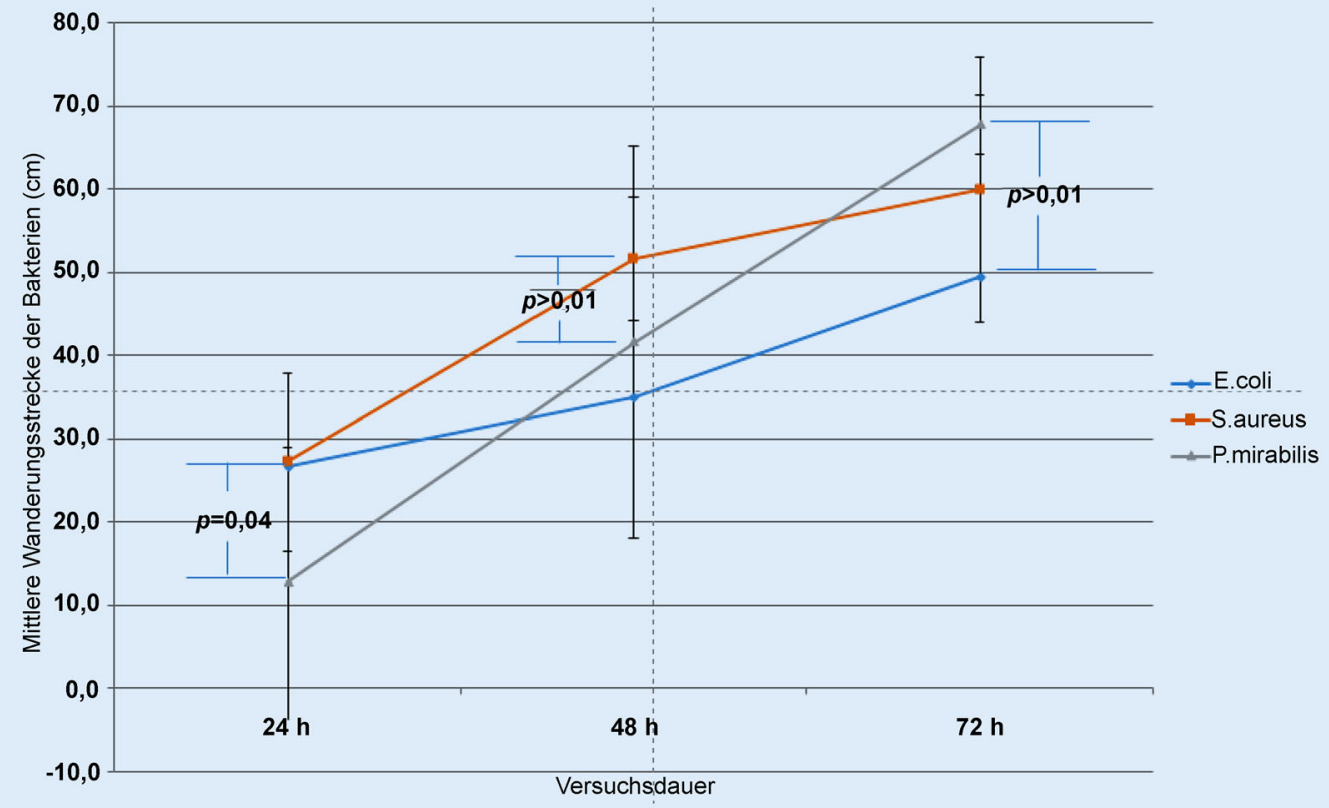

Abb. $2<$ Die mittlere Wanderungsstrecke in $\mathrm{cm}$ nach 24, 48 und $72 \mathrm{~h}$ von E. coli, S. aureus und $P$. mirabilis: Angegeben sind die Mittelwerte und Standardabweichung aus je 9 Versuchen

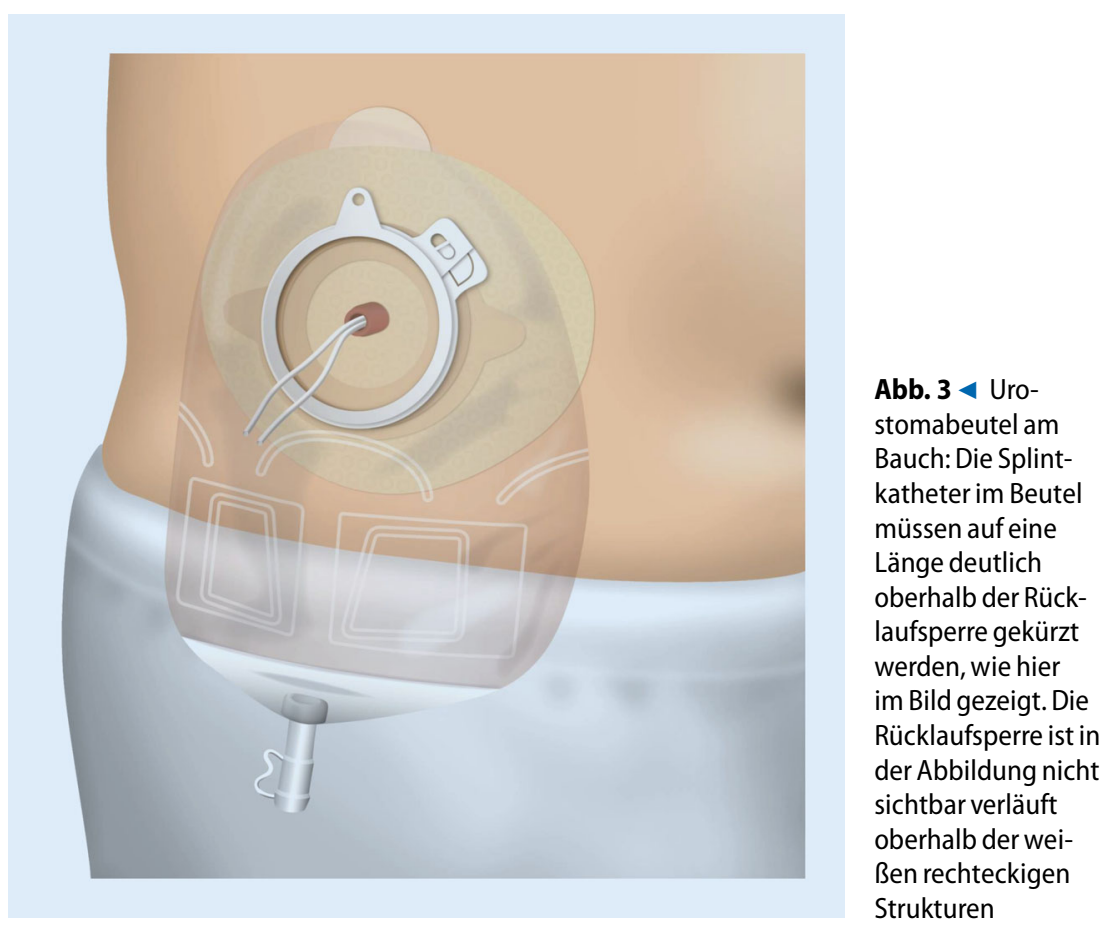

Eine effektive Infektionsprävention und ein schnelles Erkennen und Behandeln einer sich entwickelnden Infektion sind wichtig. Unsere Ergebnisse stützen besonders den Infektionspräventionsgedanken, der zum Grundsatz hat, jeglichen zusätzlichen vermeidbaren Keimeintrag auch in bereits keimbelastete Areale zu vermeiden. In diesem Fall das in der Praxis durchaus zu beobach-

\section{Schlussfolgerung}

die aufsteigenden Bakterien durch die ca. 30-40 cm langen Splintkatheter die Nieren erreichen.

\section{Fazit für die Praxis}

Bei einer bakteriellen Kontamination des aufgefangenen Urins können aufgrund der Wanderungsgeschwindigkeit der Bakterien diese nach wenigen Tagen über den Ureter in die Nieren aufsteigen. Die nicht unübliche Praxis, die Rücklaufsperre aufgrund der besseren Fixierung der Splinte zu durchstoßen, muss daher unbedingt vermieden werden (॰ Abb. 3).

\section{Korrespondenzadresse}

\section{Dr. H. Braunwarth}

Coloplast GmbH

Kuehnstraße 75, 22045 Hamburg, Deutschland dedbr@coloplast.com

\section{Einhaltung ethischer Richtlinien}

Bakterien können in harnableiteten Systemen bis $\mathrm{zu} 1 \mathrm{~cm} / \mathrm{h}$ voranwachsen. Es muss davon ausgegangen werden, dass innerhalb der vorgesehenen Liegedauer eines Splintkatheters von 10-14 Tagen,
Interessenkonflikt. F. H. H. Brill, J. Steinmann und W. Droste geben an, dass kein Interessenkonflikt besteht. H. Braunwarth und D. Hegeholz sind Angestellte der Fa. Coloplast $\mathrm{GmbH}$.

Dieser Beitrag beinhaltet keine von den Autoren durchgeführten Studien an Menschen oder Tieren. 
Open Access. Dieser Artikel wird unter der Creative Commons Namensnennung 4.0 International Lizenz (http://creativecommons.org/licenses/by/4.0/deed. de) veröffentlicht, welche die Nutzung, Vervielfältigung, Bearbeitung, Verbreitung und Wiedergabe in jeglichem Medium und Format erlaubt, sofern Sie den/die ursprünglichen Autor(en) und die Quelle ordnungsgemäßnennen, einen Link zur Creative Commons Lizenz beifügen und angeben, ob Änderungen vorgenommen wurden.

\section{Literatur}

1. EN 1616. Synthetischer Urin. 25,0 g/l Harnstoff; 9,0 g/l Natriumchlorid; 2,5 g/l Kaliumhydrogenphosphat; $2,5 \mathrm{~g} / \mathrm{l}$ wasserfreies Dinatriumhydrogenorthophosphat; 3,0 g/l Ammoniumchlorid; 2,0 g/l Kreatinin; 3,0 g/l Natriumsulfit in destilliertem Wasser. Der pH-Wert beträgt ca. 6,6

2. Feil-Peter H (2001) Stomapflege: Enterostomatherapie; Stoma-und Wundversorgung, 7. Aufl. Schlütersche $\mathrm{GmbH}$, Hannover

3. http://nurses.uroweb.org/wp-content/uploads/ EAUN_IU_Guidelines_EN_2009_LR.pdf.Zugegriffen: 4.11.2016

4. http://www.urologielehrbuch.de/harnwegs infektion_ursachen.html.Zugegriffen:4.11.2016

5. http://www.urologielehrbuch.de/ureterokutane ostomie.html.Zugegriffen:4.11.2016

6. Kommission für Krankenhaushygiene und Infektionsprävention (KRINKO) (2015) Prävention und
Kontrolle Katheter-assoziierter Harnwegsinfektionen. Bundesgesundheitsblatt Gesundheitsforschung Gesundheitsschutz 58:641-650

7. Kommission für Krankenhaushygiene und Infektionsprävention (KRINKO) (2010) Infektionsprävention in Heimen. Empfehlung der Kommission für Krankenhaushygiene und Infektionsprävention beim Robert Koch-Institut (RKI). Bundesgesundheitsblatt Gesundheitsforschung Gesundheitsschutz 48(9):1061-1080

8. Liedel B (2007) Drainage des Harntraktes. In: Jocham D, Miller K (Hrsg) Praxis der Urologie. Thieme, Stuttgart, S300

9. Mahoney M, Baxter K, Burgess J et al (2013) Procedure for obtaining a urine sample from a urostomy, ileal conduit, and colon conduit: a best practice guideline for clinicians. J Wound Ostomy Continence Nurs 40(3):277-279 (quizE1-E2)

10. Nickel JC, Downey J, Costerton JW (1992) Movement of pseudomonas aeruginosa along catheter surfaces. A mechanism in pathogenesis of catheter-associated infection. Urology 39:93-99

11. Stein $R$, Hohenfellner $M$, Pahernik $S$, Roth $S$ Thüroff JW, Rübben H (2012) Urinary diversionapproaches and consequences. Dtsch Ärztebl Int 109(38):617-622

12. Thulin H, Steineck G, Kreicbergs U et al (2010) Hygiene and urinary tract infections after cystectomy in 452 Swedish survivors of bladder cancer. BJU Int 105(8):1107-1117

\section{e.Curriculum}

\section{Akutes Nierenversagen - Interaktive Fälle zur aktuellen KDIGO-Leitlinie}

So nehmen Sie teil:

1. Anmelden: Bitte melden Sie sich mit Ihrem SpringerMedizin-Account an. Sollten Sie noch nicht angemeldet sein, können Sie sich hier registrieren: https://registrierung.springer-medizin.de/

2. Kurs auswählen und bearbeiten: Mit „Kurs starten“ können Sie die Fortbildung bearbeiten und die MC-Fragen des Fragebogens beantworten.

3. CME-Punkte sammeln: Mit 70\% richtig beantworteten Fragen haben Sie bestanden. Fachärzten werden die Punkte an den EIV übermittelt.

\section{Springer Medizin}

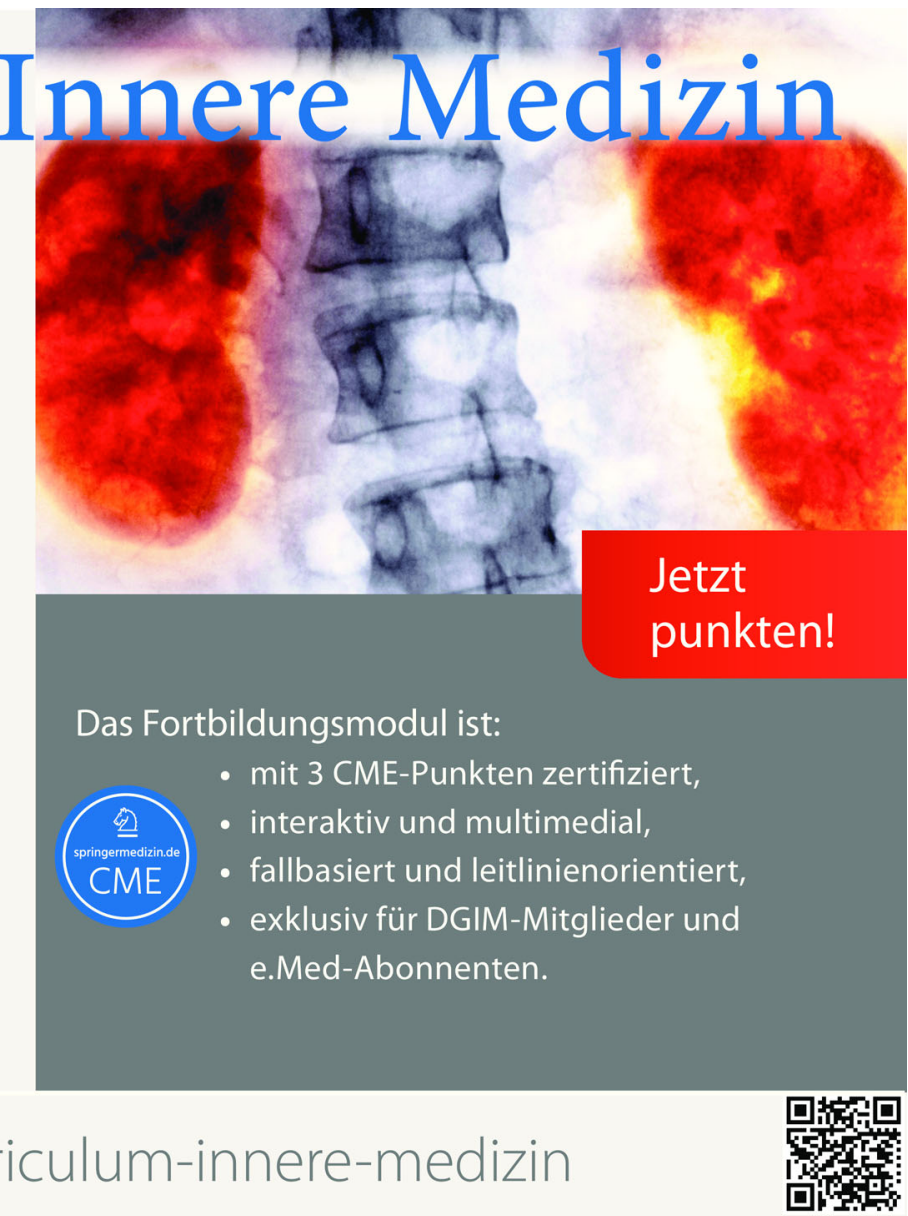

